\title{
Gradhiva
}

GRADHIV

Revue d'anthropologie et d'histoire des arts

28 | 2018

Capsules temporelles

\section{Ji Zhe, Religion, modernité et temporalité. Une sociologie du bouddhisme chan contemporain}

Paris, CNRS Éditions, coll. « CNRS Alpha », 2016

Frédéric Keck

\section{OpenEdition}

\section{Journals}

Édition électronique

URL : http://journals.openedition.org/gradhiva/3965

DOI : $10.4000 /$ gradhiva.3965

ISSN : 1760-849X

\section{Éditeur}

Musée du quai Branly Jacques Chirac

Édition imprimée

Date de publication : 28 novembre 2018

Pagination : 244-245

ISBN : $978-2-35744-111-8$

ISSN : 0764-8928

Référence électronique

Frédéric Keck, « Ji Zhe, Religion, modernité et temporalité. Une sociologie du bouddhisme chan

contemporain », Gradhiva [En ligne], 28 | 2018, mis en ligne le 28 novembre 2018, consulté le 07 janvier 2021. URL : http://journals.openedition.org/gradhiva/3965 ; DOI : https://doi.org/10.4000/gradhiva. 3965

Ce document a été généré automatiquement le 7 janvier 2021

(c) musée du quai Branly 


\section{Ji Zhe, Religion, modernité et temporalité. Une sociologie du bouddhisme chan contemporain}

Paris, CNRS Éditions, coll. « CNRS Alpha », 2016

Frédéric Keck

\section{RÉFÉRENCE}

Ji Zhe, Religion, modernité et temporalité. Une sociologie du bouddhisme chan contemporain. Paris, CNRS Éditions, coll. « CNRS Alpha », 2016, 348 p. 


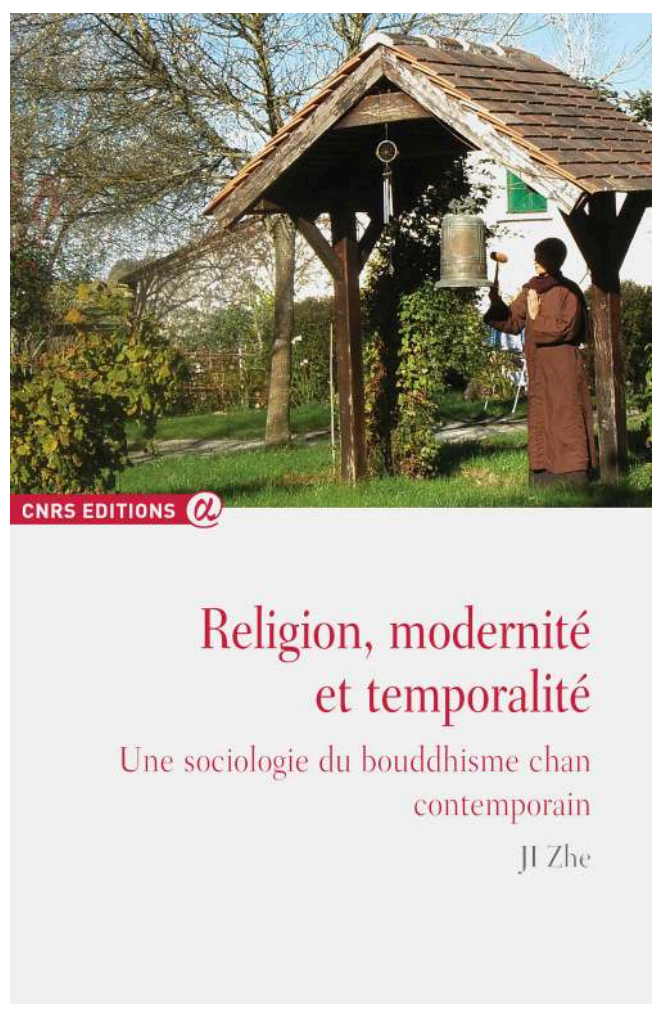

Dans le renouveau actuel des études sur les religions chinoises contemporaines ${ }^{1}$, le livre de Ji Zhe se distingue à la fois par son objet (le bouddhisme chan, version chinoise du zen japonais) et par son auteur. Professeur à l'Inalco, Ji Zhe est un intellectuel chinois soucieux d'interroger la place de la Chine dans la modernité, et il utilise pour cela à la fois l'enquête ethnographique et la théorie sociologique. Ce livre est en effet issu d'observations et d'entretiens menés sur trois sites fondés dans les années 1980 dans des aires géographiques différentes: le temple Chan Bailin au Hebei, en Chine, reconstruit sous le régime post-maoïste; la Société Chan Moderne, à Taipei ; et le Village des Pruniers, un centre bouddhique international fondé par un moine zen vietnamien en Dordogne. Il propose d'analyser les temporalités à l'œuvre dans le renouvellement du bouddhisme.

Le livre s'ouvre par une discussion des théories de la sécularisation qui décrivent la modernité comme un processus graduel de disparition et de remplacement de la religion. Si cette théorie a été formulée pour l'Europe, elle semble s'appliquer à la Chine, où le régime maoïste s'est construit sur la négation des trois religions traditionnelles (confucianisme, taoïsme et bouddhisme) en reprenant à l'Occident, via le Japon, le concept de religion (jiao). Ji Zhe s'appuie sur les principes et les pratiques du bouddhisme pour contester la conception linéaire du temps comme progrès et pour dégager une autre forme du temps que le temps cyclique de la tradition auquel il est souvent opposé. Lorsqu'il est arrivé d'Inde en Chine il y a un millénaire, le bouddhisme s'est trouvé une place entre deux religions plus anciennes, le confucianisme (plus lettré) et le taoïsme (plus populaire) en introduisant la temporalité du dharma (en chinois mofa ou « loi bouddhiste »), comptée à partir de la mort de Bouddha Sâkyamuni. Or le renouveau du bouddhisme chan en Chine depuis la fin du maoïsme tient justement, selon Ji Zhe, à ce qu'il est parvenu à insérer cette temporalité dans les pratiques ordinaires des Chinois. À rebours d'une vie sociale de plus en plus accélérée et dispersée, il propose des exercices de ralentissement et de concentration. 
3 Les enquêtes confirment ce diagnostic en observant la vie quotidienne des fondateurs $\mathrm{du}$ renouveau bouddhiste et de leurs disciples. Au temple Chan Bailin, reconstruit depuis les années 1980 dans un style chinois traditionnel, la vie des moines tourne autour de vieux cyprès qui ont échappé à la destruction. Outre qu'ils justifient le nom du temple (bailin signifie "forêt de cyprès »), ces arbres rappellent aux disciples une parabole (gong'an) de maître Zhaozhou qui répond aux interrogations les plus diverses : «Le cyprès est dans la cour. » Le cyprès devient ainsi l'objet dans lequel, selon Ji Zhe, l'histoire du temple est "encodée ", c'est-à-dire que divers enseignements s'y catalysent comme un élément signifiant du paysage. Une analyse similaire est faite à propos d'un autre gong'an de maître Zhaozhou dans lequel celui-ci répète « Va boire du thé !» Il ne s'agit pas seulement d'un monument qui rattache le présent au passé, mais d'un objet de la vie quotidienne qui brise la temporalité utilitaire du présent pour ouvrir à celle du dharma. Le fondateur du nouveau temple Chan Beilin, Jinghui, définit ainsi le chan vivant comme "une méthode pour appliquer le bouddhisme dans ce monde ».

4 Le deuxième terrain, le village bouddhiste établi en Dordogne par le moine vietnamien Thích Nhâ't Hanh, permet de comprendre comment cette conception de la temporalité agit dans la vie des disciples, y compris non-chinois. Le Village des Pruniers est un lieu où les gens viennent pour apprendre à méditer en maîtrisant leur respiration et se rencontrent pour discuter des contenus du bouddhisme, selon une forme proche du pèlerinage tel qu'il est conceptualisé par Danièle Hervieu-Léger. Ici ce n'est pas le cyprès mais la cloche qui fait basculer d'une temporalité à une autre : « La cloche est la voix du Bouddha qui nous ramène à notre pleine conscience, ici et maintenant, dit Thích Nhâ't Hanh. Chaque fois que vous entendez le son de la cloche, cessez de parler et arrêtez toute activité. » (p. 128)

5 Le troisième site d'observation est la Société Chan Moderne fondée par le bouddhiste laïc Li Yuansong à la fin des années 1980 dans la capitale de Taïwan, Taipei. C'est une communauté de laïcs en contexte urbain qui tente de résister à l'institutionnalisation en maintenant de fortes relations interpersonnelles, au point que Li Yuansong dut dissoudre la communauté en 1994 pour la refonder. Ji Zhe rattache l'existence de bouddhistes laïcs (jushi) à un texte publié en 1632, Notes de lampe dérivée des bouddhistes laïcs (jushi fendenglu), dans lequel le mot « lampe » désigne la transmission de la sagesse bouddhiste de maître à disciple. Il s'appuie sur la théorie du charisme chez Max Weber pour distinguer le virtuose, capable de répéter les gestes traditionnels de façon admirable, et le prophète, qui ouvre un nouvel avenir et suscite l'espoir d'un monde meilleur.

6 Ces derniers chapitres sur le charisme laissent peut-être échapper ce qu'il y avait de plus original dans la conception de la temporalité élaborée par Ji Zhe. En discutant les analyses wébériennes sur le passage d'une éthique extra-mondaine à une éthique intramondaine, Ji Zhe reconduit apparemment le schéma de la sécularisation qu'il cherchait à écarter. Pour saisir comment une temporalité radicalement hétérogène s'inscrit dans la vie quotidienne à travers des objets, produisant ainsi ce que Ji Zhe appelle à la suite de Pierre Bourdieu un "capital symbolique», il aurait pu reprendre les analyses d'Émile Durkheim sur le totémisme australien dans Les Formes élémentaires de la vie religieuse décrivant les " choses sociales » comme des « choses qui durent ». Chacun des sites observés par Ji Zhe se concentre en effet dans un objet qui en condense la temporalité : le cyprès, la cloche et la lampe. Mais on peut aussi considérer que le 
charisme transforme les personnes en choses, notamment par les effets qu'elles produisent après la mort : c'est peut-être une des significations de la « lampe » dans le bouddhisme laïc. La sociologie «temporaliste » proposée par Ji Zhe, visant à examiner « les conditions et les modes par lesquelles l'objet étudié adopte une forme temporelle précise » (p.292), pose de telles questions générales à travers les cas particuliers du bouddhisme chan.

\section{NOTES}

1. Voir Adam Yuet Chau (éd.), Religion in Contemporary China. Revitalization and Innovation. Londres, Routledge, 2011 ; Vincent Goossaert et David Palmer, La Question religieuse en Chine. Paris, CNRS Éditions, 2012 ; Sébastien Billioud et Joël Thoraval, Le Sage et le Peuple. Le Renouveau confucéen en Chine. Paris, CNRS Éditions, 2014.

\section{AUTEURS}

\section{FRÉDÉRIC KECK}

frederic.keck[at]college-de-france.fr 